\title{
Modelando la gestión e incubación del conocimiento
}

\author{
Cruz García Liriosa
}

Resumen - El objetivo del presente trabajo es modelar los ejes y temas centrales de la agenda para evidenciar la gestión e incubación del talento. Se realizó un estudio documental con una selección de fuentes indexadas a los principales repositorios latinoamericanos. Se contrastó un modelo estructural $\int_{\chi 2}=13,24(\mid 2 \mathrm{gl}) \mathrm{p}>, 05$; $\mathrm{CFI}=$, 997; NFI =, 990; RMSEA =, $008 \mathrm{~J}$ se establecieron dos ejes de categorías relativas a la gestión y la incubación de talentos con respecto a una selección de cinco resúmenes. En relación con el estado del conocimiento se discuten los límites y alcances en una propuesta híbrida.

Palabras clave - Formación, Gestión, Incubación, Modelo, Talentos.

Abstract - The objective of this work is to model the axes and central themes of the agenda to show the management and incubation of talent. A documentary study was carried out with a selection of sources indexed to the main Latin American repositories. A structural model $\int_{\chi 2}=13.24(\mid 2 \mathrm{gl}) \mathrm{p}>.05$ was contrasted; $\mathrm{CFI}=$ .997; NFI =, 990; RMSEA $=.008 \mathrm{~J}$ Two category axes relating to talent management and incubation were established with respect to a selection of five abstracts. In relation to the state of knowledge, the limits and scope are discussed in a hybrid proposal.

Keywords - Institutionalism, Agenda, Entrepreneurship, Talents, Training.

${ }^{a}$ Universidad Autónoma del Estado de México, México. E-mail: garcialirios@icloud.com

\section{CÓMO CITAR} HOW TO CITE:

García Lirios, C. (2022)

Modelando la gestión e incubación del talento. Interconectando Saberes, (I3), 59-66.

https://doi.org// 0.25009/is.v0il3 .2705

Recibido: 25 de mayo de 2021 Aceptado: 22 de noviembre de 2021

Publicado: 31 de enero de 2022 


\section{INTRODUCCIÓN}

El índice de Desempeño en Ciencia y Tecnología de la Organización para la Cooperación y el Desarrollo reporta un mayor incremento de co-patentes entre universidades $y$ organizaciones con fines de lucro durante la pandemia (García, 2020). En contraste, los proyectos de emprendimiento público y universitario se encuentran más rezagados (véase Figura I).

\section{Figura I}

Desempeño en Ciencia y Tecnología

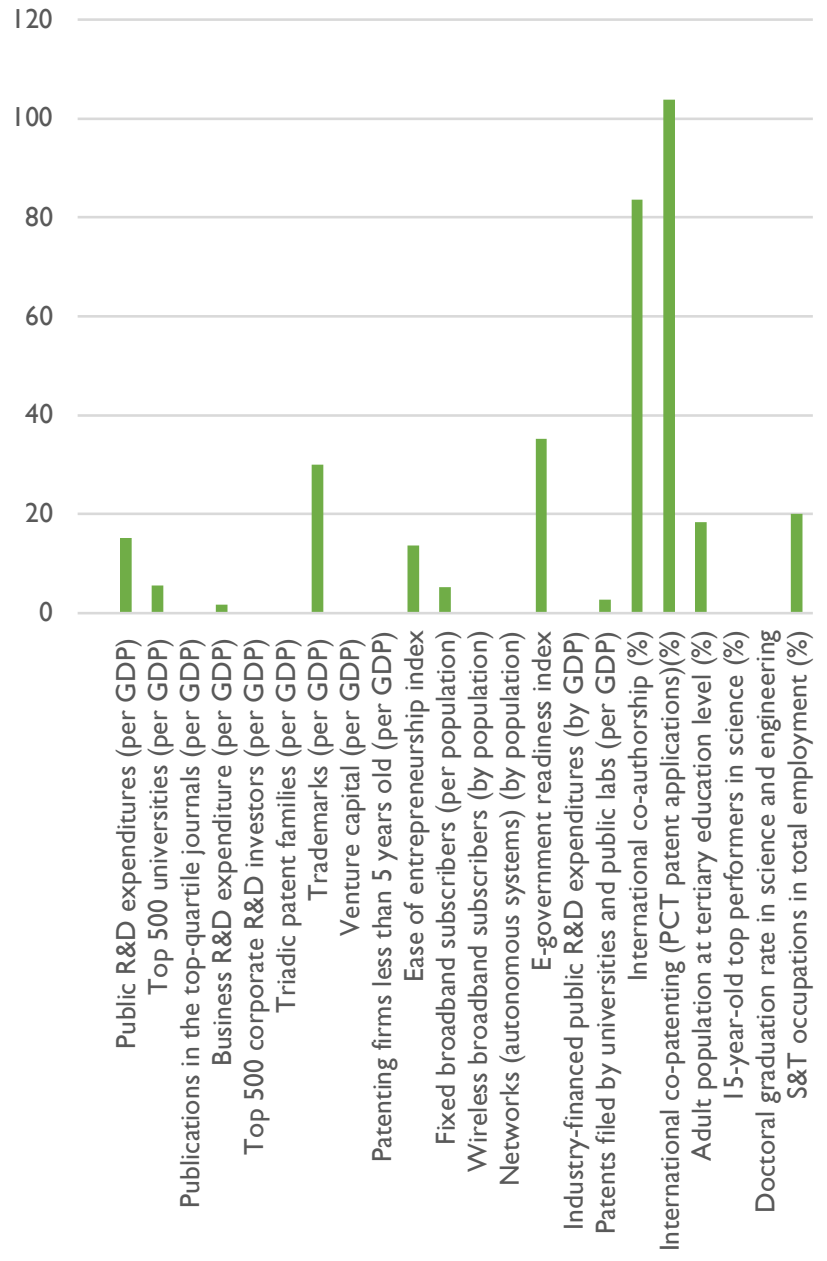

Nota: Con información de la Organización para la Cooperación y el Desarrollo (202I).
El gobierno electrónico, indicador del índice de Desempeño en Ciencia y Tecnología, sugiere que la gestión y la incubación de talentos está orientada hacia la producción de patentes más que hacia la transferencia de conocimiento y su reproducción en alianzas estratégicas entre universidades públicas (Lukosiute et al., 2019). Por consiguiente, el estudio de ambos indicadores, gestión e incubación de proyectos emprendedores suponen la discusión en torno a la formación del capital humano, enfatizando la relación entre la academia, profesión y trabajo (Aguilar et al., 2020).

De este modo, la gestión del conocimiento alude a los sistemas de codificación de relaciones de confianza, expectativas y saberes orientadas por objetivos, tareas y metas comunes (Van Hoek et al., 2020). Se trata de una red académica, profesional o laboral que permite el desarrollo de innovaciones en procesos y productos evaluables y comparables (García et al., 202I). En términos de revisión sistemática, la gestión del conocimiento es la convergencia de indicadores relativos a hallazgos explicativos $y$ predictivos de las diferencias y similitudes entre las partes gestoras, productoras y traductoras de datos (García, 2019).

De este modo, la incubación de proyectos es un indicador de la gestión del conocimiento. Se trata de la emergencia de proyectos emprendedores en situaciones de riesgo inminente o contingencias imprevistas (Quiroz \& García, 2021). La incubación de proyectos sugiere que la gestión del conocimiento se ha diseminado a través de un clima de confianza, relaciones e innovaciones más que de tareas (Sánchez et al., 2020). Por consiguiente, la formación del capital humano, principalmente el capital 
intelectual se establece desde la confianza en la gestión y la tecnología (Carreón et al., 2017).

No obstante que los indicadores de gestión del conocimiento pueden ser observables en la incubación de proyectos, estos pueden ser inferidos desde la literatura publicada desde diciembre de 2019 a junio de 2021. En este sentido, la revisión de hallazgos puede generar una base de datos suficiente para ser calificada por jueces expertos y modelada por ecuaciones estructurales con mínimos cuadrados no ponderados ordinarios (Hassan, 2020). Esto es así porque los resúmenes de las publicaciones recuperan la información relativa a la gestión del conocimiento y los indicadores de la incubación de proyectos (Pérez et al., 2018).

Es así como el objetivo del presente trabajo fue modelar las relaciones entre las categorías de la gestión del conocimiento, la incubación de proyectos y sus indicadores reportados en los resúmenes de la literatura publicada de 2019 a 2021 , considerando la evaluación de expertos en rondas de calificación, retroalimentación y reconsideración.

¿Existirán diferencias significativas entre la estructura teórica de la gestión del conocimiento con respecto a las calificaciones de jueces expertos en la temática?

Las premisas que guían el presente trabajo sugieren el confinamiento y distanciamiento de personas, estrategias de mitigación de la pandemia, incidieron sobre la gestión del conocimiento en las universidades públicas de un modo tal que redujo las incubaciones de proyectos (Fierro et al., 2018). Las partes involucradas, gestores y talentos debieron orientar sus objetivos, tareas y metas hacia la codificación de saberes, aminorando la confianza en liderazgos y exacerbando las tareas de reproducción del conocimiento (García et al., 2016). A medida que la pandemia se prolonga, la gestión del conocimiento se generó sólo en las alianzas estratégicas entre organizaciones con fines de lucro, desplazando a las universidades de la gestión (Espinoza et al., 202I). Las universidades públicas produjeron un conocimiento orientado hacia la optimización de recursos más que hacia la innovación de procesos, registrándose esta prevalencia en la literatura seleccionada (Sánchez et al., 2017).

\section{MÉTODO}

Diseño. Dado que los estudios relacionados con la gestión e incubación de proyectos abordan la necesidad y procesamiento de la información, se realizó una investigación documental, retrospectiva y comparativa con una selección de fuentes indexadas a repositorios internacionales: Academia, Copernicus, Dialnet, Frontiers, Latindex, Redalyc, Scielo, Scopus y Zenodo, considerando las palabras clave "gestión", “incubación” y "talento“ (véase Tabla I).

\section{Tabla I}

Descriptivos de la muestra

\begin{tabular}{ccccccc}
\hline Repositorio & \multicolumn{3}{c}{ Gestión } & \multicolumn{3}{c}{ Incubación } \\
\hline & 2019 & 2020 & 2021 & 2019 & 2020 & 2021 \\
\hline Academia & 3 & 2 & 2 & 3 & 4 & 2 \\
\hline Copernicus & 2 & $\mathrm{I}$ & 4 & 2 & 3 & $\mathrm{I}$ \\
\hline Dialnet & 4 & $\mathrm{I}$ & 3 & 2 & 2 & 4 \\
\hline Ebsco & 5 & 3 & 2 & $\mathrm{I}$ & 5 & 5 \\
\hline Frontiers & 3 & 2 & $\mathrm{I}$ & 3 & 4 & 3 \\
\hline Latindex & 2 & 4 & 2 & 4 & 3 & 4 \\
\hline Redalyc & $\mathrm{I}$ & 3 & 3 & 2 & $\mathrm{I}$ & 2 \\
\hline Scielo & 3 & $\mathrm{I}$ & 2 & 3 & 2 & 3 \\
\hline Scopus & 2 & 3 & 4 & 2 & $\mathrm{I}$ & 2 \\
\hline Zenodo & 4 & 2 & $\mathrm{I}$ & $\mathrm{I}$ & $\mathrm{I}$ & $\mathrm{I}$ \\
\hline
\end{tabular}

Nota: Elaborada con los datos del estudio. Se incluyen las frecuencias de la búsqueda de información mediante el uso de palabras claves en el periodo de diciembre de 2019 a junio de 2021 . 
Muestra. Se realizó una selección de resúmenes, considerando la relación entre la gestión del talento y la incubación durante la pandemia para poder evaluar sus contenidos mediante la técnica Delphi (véase Tabla 2).

\section{Tabla 2}

Descriptivos de la muestra

\begin{tabular}{|c|c|c|c|c|c|}
\hline $\begin{array}{c}\text { Extrac } \\
\text { to }\end{array}$ & $\begin{array}{l}\text { Reposito } \\
\text { rio }\end{array}$ & $\underset{r}{\text { Auto }}$ & $\begin{array}{c}\text { Añ } \\
0\end{array}$ & $\begin{array}{c}\text { Referen } \\
\text { cias }\end{array}$ & $\begin{array}{c}\text { Modelami } \\
\text { ento }\end{array}$ \\
\hline I & Academia & $\begin{array}{l}\text { Aguil } \\
\text { ar et } \\
\text { al., }\end{array}$ & $\begin{array}{c}202 \\
0\end{array}$ & 43 & $\begin{array}{l}\text { Gestión } \rightarrow \\
\text { Formación }\end{array}$ \\
\hline 2 & $\begin{array}{l}\text { Copernic } \\
\text { us }\end{array}$ & $\begin{array}{l}\text { Sánch } \\
\text { ez et } \\
\text { al., }\end{array}$ & $\begin{array}{c}202 \\
0\end{array}$ & 23 & $\begin{array}{l}\text { Gestión } \rightarrow \\
\text { Formación }\end{array}$ \\
\hline 3 & Dialnet & $\begin{array}{c}\text { Garcí } \\
\text { a }\end{array}$ & $\begin{array}{c}201 \\
9\end{array}$ & 36 & $\begin{array}{l}\text { Gestión } \rightarrow \\
\text { Formación }\end{array}$ \\
\hline 4 & Ebsco & $\begin{array}{l}\text { Quir } \\
\text { oz \& } \\
\text { Garcí } \\
\text { a }\end{array}$ & $\begin{array}{c}202 \\
1\end{array}$ & 33 & $\begin{array}{c}\text { Gestión } \rightarrow \\
\text { Emprendimi } \\
\text { ento }\end{array}$ \\
\hline 5 & Frontiers & $\begin{array}{c}\text { Garcí } \\
\text { a et } \\
\text { al., }\end{array}$ & $\begin{array}{c}202 \\
1\end{array}$ & 25 & $\begin{array}{c}\text { Gestión } \rightarrow \\
\text { Adiestramie } \\
\text { nto }\end{array}$ \\
\hline
\end{tabular}

Nota: Fuente: Elaborada con los datos del estudio; $\leftarrow$ relación formativa $\rightarrow$ relación reflejante. Se incluyen los resúmenes seleccionados considerando el tipo de modelamiento al relacionar la gestión del conocimiento con la incubación de proyectos.

Proceso. La técnica Delphi se utilizó con jueces expertos en gestión e incubación del talento durante tres rondas de análisis: a) Calificación donde se asignó un valor de - I para la gestión e incubación de talentos en riesgo $y+I$ para la vinculación en situación post pandemia; b) Retroalimentación al comparar las calificaciones con el promedio; c) reconsideración ahora el juez emitió una nueva calificación, o reiteró su criterio (Bustos et al., 2019).

Análisis. Los datos fueron procesados en el paquete de análisis estadístico para ciencias sociales (SPSS 20), así como en el software NetMiner versión 3.0 y Amos 4.0, considerando los parámetros de distribución normal no paramétrica, contingencia, proporción de probabilidades, ajuste y residual (Hernández et al., 2020).

\section{RESULTADOS}

Los valores alcanzaron los requisitos mínimos de distribución normal, así como las estadísticas de relación de contingencia para contrastar la hipótesis de diferencias significativas y los parámetros de razón de probabilidad que establecen los umbrales de riesgo (véase Tabla 3). Es decir, el inventario Delphi que registra las calificaciones de los jueces expertos en la gestión del conocimiento cumple con los requerimientos para el modelamiento de las relaciones entre las categorías y los hallazgos reportados en la literatura seleccionada. Este cumplimiento radica en la distribución normal de las calificaciones en las tres rondas evaluativa, retroalimentativa y reconsiderativa.

Una vez establecidas las relaciones de contingencia entre las categorías de gestión e incubación del talento, así como los umbrales de riesgo percibidos por los jueces expertos en la materia, se procedió a observar la estructura de ejes, trayectorias y relaciones entre los elementos con el propósito de anticipar escenarios de riesgo (véase Figura 2). La estructura resultante sugiere que la gestión del conocimiento es concomitante con la incubación de proyectos. Es decir, en la formación del capital humano, la traducción y codificación de información estaría relacionada con el emprendimiento, la optimización y la innovación de procesos y productos. 
Modelando la gestión e incubación del talento

Tabla 3

Descriptivos del instrumento

\begin{tabular}{|c|c|c|c|c|c|c|c|}
\hline $\mathbf{E}$ & M & DE & el & e2 & e3 & e4 & e5 \\
\hline \multicolumn{8}{|l|}{ RI } \\
\hline el & ,659 &, 135 & & & & & \\
\hline e2 & 672 & , I78 & ,46 8,2।,49) & & & & \\
\hline e3 &, 562 &, 109 & ,54 $(, 32,67)$ & ,54 (,24,59) & & & \\
\hline e4 & 674 &, 143 & ,56 (,34,78) & ,52(,24,78) & ,46 (,2। I,58) & & \\
\hline e5 & ,782 &, 172 & ,57 $(, 21,58)$ & ,43 $(, 29,76)$ & $32(, 26,58)$ & , $21(, 18,43)$ & \\
\hline \multicolumn{8}{|l|}{ R2 } \\
\hline el & ,603 &, 135 & & & & & \\
\hline e2 & ,671 &, 121 & ,56 $(, 23,67)$ & & & & \\
\hline e3 & ,683 & , I78 & ,43 $(, 29(, 22,60)$ & ,36 (,21,67) & & & \\
\hline e4 & ,793 &, 198 &, $54(, 32,58)$ & , II $(, 10,19)$ & ,35 $(, 24,54)$ & & \\
\hline e5 & 624 &, 135 & ,65 $(, 32,68)$ & , $21(, 18,39)$ & ,35 $(, 20,44)$ & ,32 $(, 27,40)$ & \\
\hline \multicolumn{8}{|l|}{ R3 } \\
\hline el & ,650 &, 132 & & & & & \\
\hline e2 & ,635 &, 124 & ,34 (,25, 40) & & & & \\
\hline e3 & ,65I &, 165 & $32(, 21,44)$ & ,45 $(, 25,49)$ & & & \\
\hline e4 & ,698 & 190 & ,43 $(, 27,39)$ & ,32 $(, 20,46)$ & ,21 $(, 32,76)$ & & \\
\hline e5 & 624 &, 167 & ,56(,25,67) & , 37 $(, 21,50)$ & , 32 $(, 25,67)$ & ,32 (,25, 43) & \\
\hline
\end{tabular}

Nota: Elaborada con los datos del estudio. Simbología; E = extracto, el = Aguilar et al., (2020), e2 = Sánchez et al., (2020), e3 = García (20l9), e4

= Quiroz \& Garcia (202I), e5 = Garcia et al., (202I), R = Ronda, RI = Calificación, R2 = Retroalimentación, R3 = Reconsideración, M = Media,

$\mathrm{DE}=$ Desviación Estándar, $($ ) = Intervalo de confianza

\section{Figura 2}

Modelo de ecuaciones estructurales

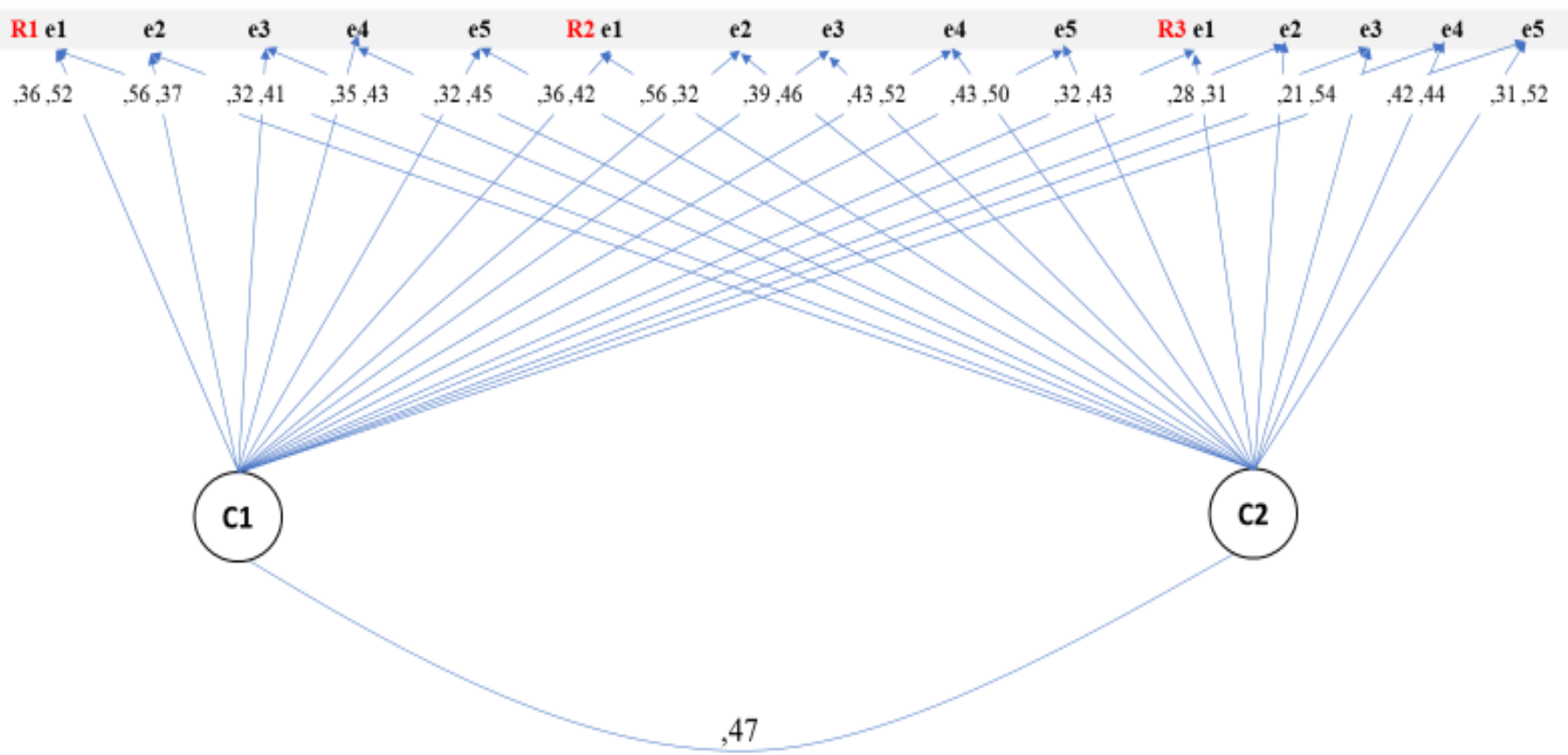

Nota: Elaborada con los datos del estudio. Simbología; E = extracto, el = Aguilar et al., (2020), e2 = Sánchez et al., (2020), e3 = García (2019), e4 = Quiroz \& Garcia (202I), e5 = Garcia et al., (202I), R = Ronda, RI = Calificación, R2 = Retroalimentación, R3 = Reconsideración, M = Media, $\mathrm{DE}=$ Desviación Estándar, $\mathrm{C}=$ categoría, $\mathrm{Cl}=$ Gestión, $\mathrm{C} 2=$ Incubación $\leftarrow$ relación formativa $\rightarrow$ relación reflejante 
La estructura resultante muestra que ambas categorías: gestión e incubación están relacionadas con las propuestas de modelado en los cinco extractos de hallazgos calificados por los jueces. Los parámetros de ajuste y residuales $\int \chi 2=13,24(12 \mathrm{gl}) \mathrm{p}>, 05 ; \mathrm{CFI}=$, 997; NFI =, 990; RMSEA =, $008 \mathrm{~J}$ sugieren la norma de la hipótesis nula relativa a las diferencias significativas entre la estructura teórica con respecto a la prueba empírica del modelo.

\section{DISCUSIÓN}

El aporte del presente trabajo al estado de la cuestión radica en el modelamiento de las categorías y los resúmenes que emergieron durante el periodo que va de diciembre de 2019 a junio de 2021 , considerando a la gestión como una codificación de saberes que pudo ser develada por las calificaciones de jueces expertos en la temática. Se encontró una estructura donde prevalece la gestión y la incubación asociadas a cinco estudios que revisaron sistemáticamente la relación.

La estructura encontrada, sugiere que ambas categorías están relacionadas entre sí y con respecto a los hallazgos seleccionados. La teoría de la gestión del conocimiento es opuesta a este resultado porque la confianza entre las partes involucradas en una alianza estratégica como la de universidades y organizaciones sugiere la transferencia de saberes más que el emprendimiento (Aguilar et al., 2019). Es decir, la gestión y la incubación están medidas por un clima de confianza entre las partes involucradas. De acuerdo con la teoría de la gestión del conocimiento está confianza hacia la tecnología propiciará la innovación de procesos porque los objetivos, tareas y metas serían orientados por los datos procesables en dispositivos.
El ajuste de la tecnología de procesamiento de información a las habilidades computacionales explicaría la innovación de procesos, aunque la teoría de la gestión sugiere que en eventos de riesgo prevalece la optimización de recursos (Aguinis y Burji, 202I). La inclusión de ambas categorías, optimización e innovación, como mediadoras entre la gestión y la incubación, anticiparía escenarios de emprendimiento. Tal modelo permitiría explicar un proceso subyacente a las crisis como lo son las oportunidades, retos $y$ desafíos. Estos indicadores de emprendimiento podrían vincularse a la gestión de saberes entre las partes involucradas. De este modo, el modelo resultante incluiría oportunidades, gestión, optimización, innovación e incubación de proyectos. Este proceso de formación del capital humano se distingue de los modelos tradicionales en donde la gestión se realiza desde la confianza hacia líderes.

La diferencia entre el modelo propuesto basado en la confianza hacia la tecnología y el modelo centrado en la confianza hacia los liderazgos radica en que el primero alcanza la innovación desde la autogestión de oportunidades (Cao et al., 2020). Mientras que el modelo de liderazgos supone una innovación desde la formación de talentos mediante una transferencia de conocimientos. Ambos modelos pueden ser contrastados a fin de poder obtener un híbrido que explique y anticipe la gestión del conocimiento en situaciones de riesgo, pero la técnica Delphi asociada a los mínimos cuadrados no ponderados ordinarios serían insuficientes. Es necesario una minería de calificaciones de expertos para modelar las variables. 


\section{CONCLUSIONES}

La gestión del conocimiento es un eje central en la agenda investigativa publicada de diciembre de 2019 a junio de 2020 , considerando la relación que guarda con la incubación de proyectos. A diferencia del reporte de la OCDE (202I) donde la incubación se realiza entre organizaciones con fines de lucro o en alianza con universidades, el modelo desarrollado se orienta hacia la colaboración entre universidades públicas. De este modo, la gestión pública del conocimiento pude ser modelada con otras variables y categorías que la literatura identifica como mediadoras.

\section{REFERENCIAS}

Aguilar, J. A., Perez, M. I., Perez, C., Morales, M. L. y Garcia, C. (20/9). Gobernanza de las redes de conocimiento: Contrastación de un modelo para el estudio de la formación consensuada. Alternativas, 40 (I), 24-5I

Aguinis, H. y Burji, J. (202I). Desafíos de la gestión del talento durante Covid-1 9 y más allá: Gestión del rendimiento al rescate. Investigación empresarial trimestral, I (I), I-8

https://journals.sagepub.com/doi/pdf/10.1 I77/234 09444211009528

Bustos, J. M., Juárez, M., Bermúdez, G., Quintero, M. L., Aldana, W. I. y Garcia, C. (20I9). Cultura organizacional y desarrollo local en micro y pequeños negocios emprendedores e innovadores. Informática, 40 (I), 7I-83

Cao, Y., Shan, J., Gong, Z. y Gao, Y. (2020). Estado y desafíos de la gestión de emergencias de salud pública en Chin relacionados con Covid- 19. Frontiers in Health Public, 8 (8I), 250-256

Carreón, J., Hernández, J., Quintero, M. y García, C. (20I7). Confiabilidad y validez de un instrumento que mide la colaboración organizacional en una universidad pública de Huehuetoca, centro de México. Invurnus, 12 (2), 9- I7

Espinoza, F. Campos, L. L. y García, C. (202I). Tutorial networks in the development of the research protocol. International Journal Advances in Social Sciences, 9 (I), I-8
Fierro, E., García, C. y Delgado, M. A. (20/8).

Especificación de un modelo para el estudio de la formación profesional. Analítica, I (I), 97- 108

Garcia, C. (2019). Inteligencias y sabidurías organizacionales: Redes de conocimiento en torno al aprendizaje de la complejidad. Psicogente, 22 (4I), I-28

Garcia, C. (2020). Specification a model for study of thinking entrepreneurship. Annals of Language and Literature, 4 (2), 28-40

García, C., Carreón, J., Sánchez, A., Sandoval, F. R., y Morales, M. L. (2016). Confiabilidad y validez de un instrumento que mide el liderazgo y la gestión educativa. Eqhidad, 5 (I), |09-I 3 I

García, C., Molina, H. D. y Molina, M. R. (202I). Specification of a business training model using the virtual classroom before Covid-19. Educativa, 24 (I), 26-39

Hassan, NA (2020). Las incubadoras de empresas universitarias como herramienta para acelerar el emprendimiento: perspectiva teórica. Revisión de Economía y Ciencias Políticas, I0 (I), I-20 https://www.emerald.com/insight/content/doi/I0. | I08/REPS-|0-2019-0|42/full/pdf

Hernández, J., Carreón, J., Juárez, M. y García, C. (2020). Specifying a model for self-study. Public Security \& Public Order, 24 (I), 368-384

Lukosiute, K., Jensen, S. y Tanev, S. (2019). ¿Es siempre bueno unirse a una incubadora y aceleradora de empresas? Revisión de la gestión e innovación tecnológica, 9 (I2), 5-17 https://timreview.ca/sites/default/files/article_PDF /Lukosiute_et_al_TIMReview_July2019.pdf

Organización para la Cooperación y el Desarrollo Económicos (202I). Estadísticas por país. OCDE https://www.oecdilibrary.org/development/getting-itright 9789264292062-en

Pérez, G., García, C. y Carreón, J. (2018). Redes de conocimiento en torno al desarrollo organizacional en una universidad pública del Estado de México. Invurnus, 13 (3), 26-35

Quiroz, C. Y. y García, C. (202I). Redes de formación profesional, gestión, administración y emprendimiento del conocimiento. Revista Estrategia Organizacional, 10 (I), 20-34

Sanchez, A., Figueroa, O., Espinoza, F., Molina, H. D., Valdés, O., Fierro, E. y Garcia, C. (2020). Estructura factorial confirmatoria de la gestión del conocimiento. Alternativas, 44 (I), 53-66 
Sánchez, M., Juárez, M., Bustos, J. M., Fierro, E. y García, C. (2017). Confiabilidad y validez de una escala de gestión del conocimiento en una universidad pública del centro de México. Cuadernos Hispanoamericanos de Psicología, 17 (2), $61-70$

Van Hoek, R., Gibson, B. y Johnson, M. (2020). Gestión del talento para una cadena de suministro posterior a Covid-19. El rol crítico de los gerentes. Revista de logística empresarial, 4 (4), 334-336

https://onlinelibrary.wiley.com/doi/epdf/ I0.I I I I/j bl. 12266 